\title{
Glucagon-like-peptide 1 Receptor imaging specifically localizes insulinomas in patients with Multiple Endocrine Neoplasia Type 1 (MEN-1)
}

Kwadwo Antwi ${ }^{1}$, Melpomeni Fani ${ }^{1}$, Tobias Heye ${ }^{1}$, Guillaume Nicolas ${ }^{1}$, Elmar Merkle ${ }^{1}$, Francois Pattou ${ }^{2}$, Ashley Grossmann ${ }^{3}$, Philippe Chanson ${ }^{4}$, Jean Claude Reubi ${ }^{5}$, Beat Gloor ${ }^{6}$, Damian Wild *1, Emanuel Christ *7 *shared last authors

${ }^{1}$ Clinic of Radiology and Nuclear Medicine, University of Basel Hospital, Switzerland

2Department of endocrine surgery, Lille University Hospital, France

${ }^{3}$ Oxford Centre for Diabetes, Endocrinology and Metabolism, University of Oxford, UK

${ }^{4}$ Division of Endocrinology, Hospital Bicetre Paris XI, France

${ }^{5}$ Division of Experimental Pathology, Department of Pathology, University of Bern, Switzerland

${ }^{6}$ Department of Visceral Surgery, University Hospital of Bern, Switzerland

${ }^{7}$ Division of Diabetology, Endocrinology, and Metabolism, Inselspital Bern, University Hospital and University of Bern, Switzerland

\section{Background}

- Surgery is often the only treatment option that can effectively treat patients with insulinomas in MEN-1.

- The surgical intervention should be limited as surgery can not cure patients with MEN-1.

- It is mandatory to correctly localize insulin secreting tumors from other neuroendocrine tumors.

- Benign insulinomas express Glucagon-Like Peptide-1 Receptor (GLP-1R) in nearly $100 \%$ of the cases and at a high density.

- Targeted GLP-1R imaging has been shown to be a valid and non-invasive tool to localize theses small tumors.

- It is unknown, whether GLP-1 R imaging is useful in the context of MEN-1.

\section{Aim}

We aimed at assessing the utility of GLP-1 receptor (GLP-1R) imaging in the identification of insulinomas from other pancreatic lesions in the context of MEN-1.

\section{Methods}

6 patients with proven endogenous hyperinsulinemic hypoglycemia and neuroglycopenia in the context of MEN-1 were included.

All patients received abdominal SPECT/CT after the injection of a standard activity of ${ }^{111}$ In-DOTA-exendin-4.

Four patients underwent additional imaging with a standardized contrast media enhanced $3 \mathrm{~T} \mathrm{MRI}$ and a ${ }^{68} \mathrm{Ga}-D O T A-e x e n d i n-4$ PET/CT scan as part of the study. Standard of comparison was the histological diagnosis after surgery.

\section{Results}

- Six patients (4 females and 2 males) were included (age range 18-49 years).

- Until today 5 of 6 patients have been operated.

- Conventional imaging revealed a total of 11 suspicious pancreatic or peripancreatic lesions.

- PET/CT and SPECT/CT imaging together revealed 6 lesions with a high expression of Glucagon-like Peptide-1 receptors (GLP-1R) suspicious for an insulinoma.

- Based on the GLP-1R imaging all 6 lesion were surgically removed, histopathology confirmed the diagnosis of a benign insulinoma and all 5 patients presented with normalized blood sugar levels after sugery.

\section{Conclusion}

In MEN-1 patients, insulin secreting pancreatic NET express GLP$1 R$ in a high incidence and density.

Adding GLP-1R imaging to conventional imaging is a helpful tool in differentiating insulinomas from other pancreatic islet tumors expressed in MEN-1 patient and may guide the surgical intervention.
Figures: Ce 3T MRI, ${ }^{111}$ In-Octreoscan SPECT/CT and ${ }^{68} \mathrm{Ga}$-DOTA-exendin-4 PET/CT in one patient with MEN-1

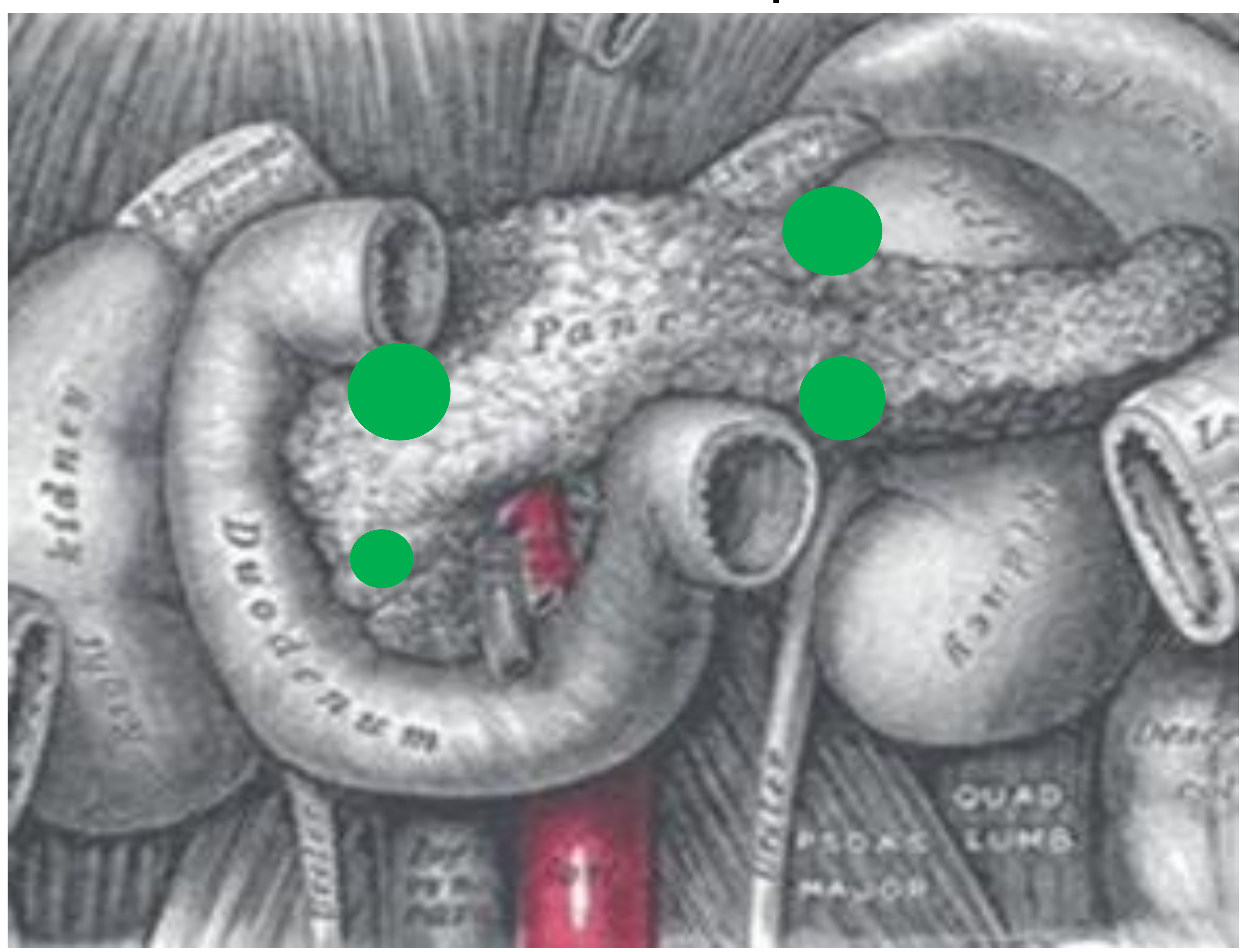

Figure 1: 3T MRI revealed 4 lesions in the pancreas.

Differentiation between insulinomas and other neuroendocrine tumors is not possible with MRI
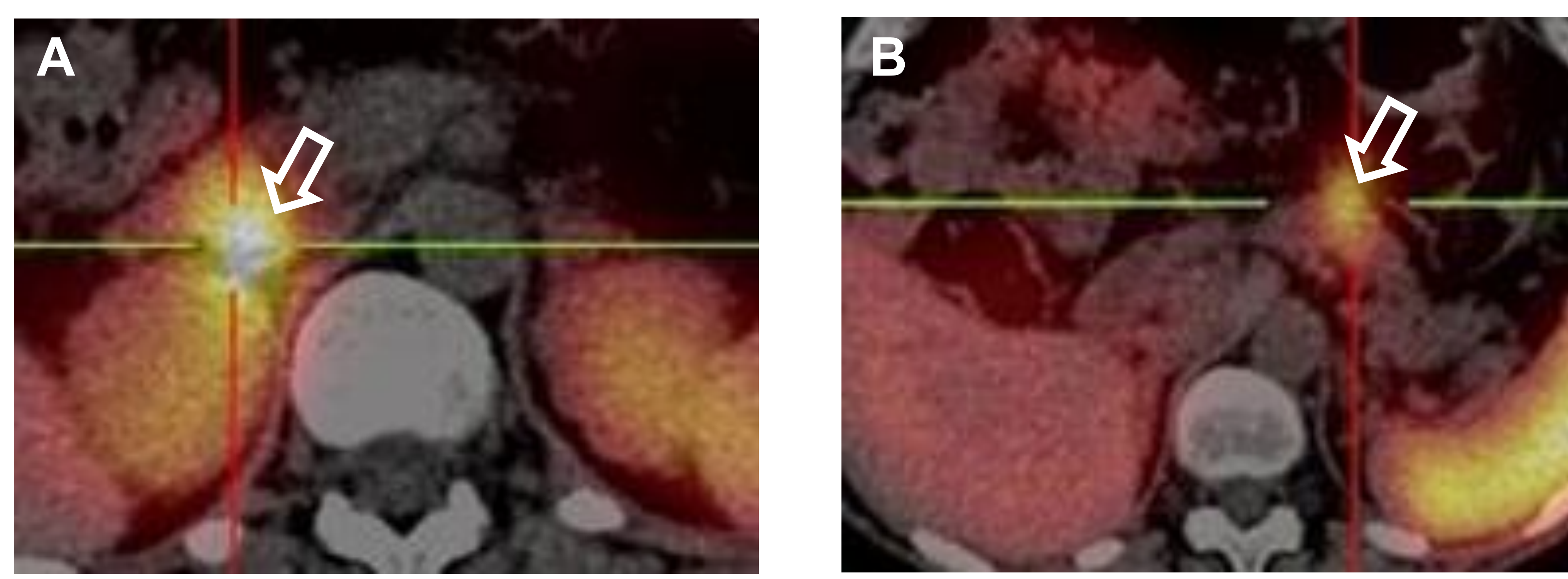

Figure 2: ${ }^{111} \mathrm{In}-$ Octreoscan SPECT/CT shows a lesion with somatostatin receptor (SSTR) expression in the cranial portion of the pancreatic head $(A)$ and another SSTR positive lesion in the ventral portion of the pancreatic tail (B). Histopathology: $(A)$ Gastrinoma (B) Ppoma
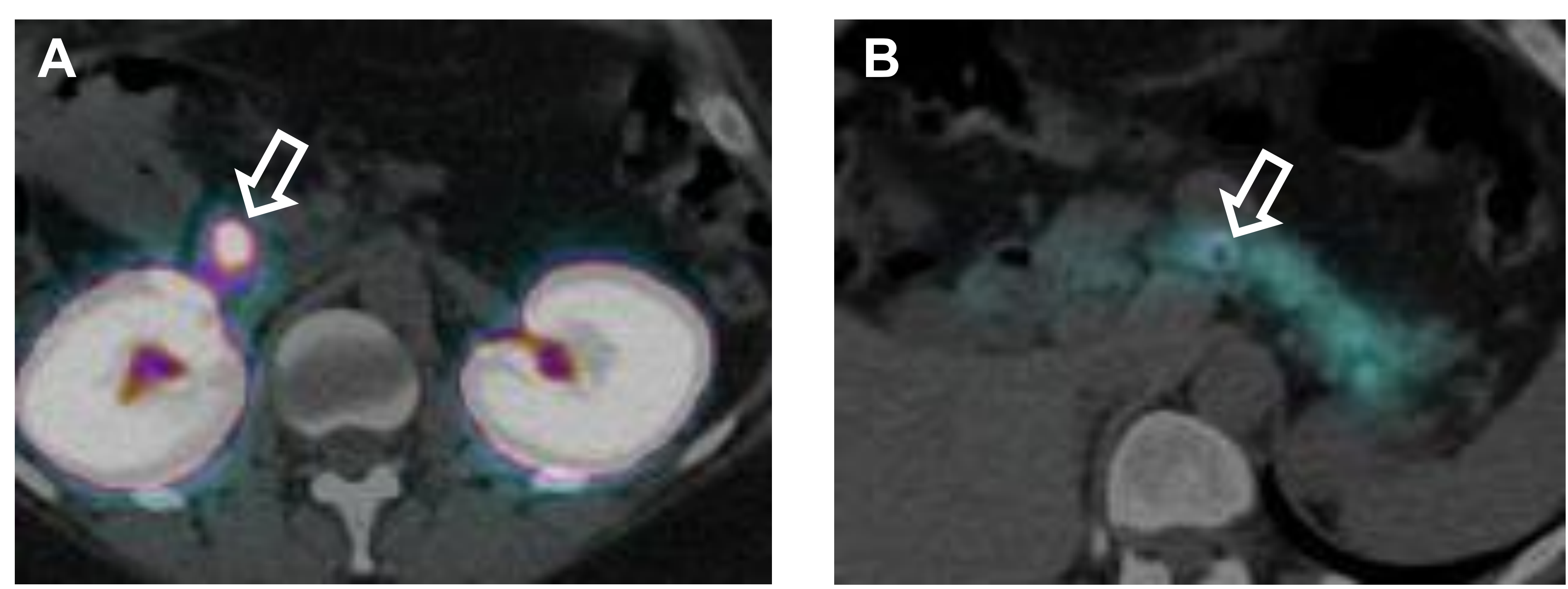

Figure 3: ${ }^{68} \mathrm{Ga}-\mathrm{DOTA}$ exendin-4 PET/CT shows a lesion with strong GLP-1R expression in the uncinate process $(A)$ and an another GLP-1R positive lesion in the dorsal portion of the pancreatic tail (B). Histopathology: (A) Insulinoma (B) Insulinoma 